\title{
Liderazgo en Empresas Familiares
}

\author{
Leadership in Family Businesses \\ Maribel Nava Pozos ${ }^{a}$, Lucero Cuatepotzo Sánchez ${ }^{b}$, Maria del Carmen Rodríguez \\ Flandes $^{c}$
}

\begin{abstract}
:
Family businesses in the last decade of the twentieth century have been marked by the tonic of a constant and progressive change due to their great participation in their economic, social and cultural dimensions, the participation and contribution these economies are increasingly complex and difficult to understand. Due to its management in the interior, however, the custom and practices that have developed have transcended for generations and remain permeated in the local and national economies in a constant manner, therefore the study of family businesses is essential in order to contribute to strengthen them and keep them actively participating in the economy.
\end{abstract}

\section{Keywords:}

Participation, patrimony, strategy, economy, development

\section{Resumen:}

Las empresas familiares en la última década del siglo XX han estado marcadas por la tónica de un cambio constante y progresivo debido a su gran participación en sus dimensiones económico, social y cultural la participación y aportación estas economías son cada vez más complejas y difícil de entender debido a su manejo al interior sin embargo la costumbre y las prácticas que se han desarrollado han trascendido durante generaciones y siguen permeado en las economías, locales y nacionales de manera constante, por lo anterior es indispensable el estudio a las empresas familiares a efecto de contribuir a su fortalecimiento y mantenerlas participando de manera activa dentro de la economía.

\section{Palabras Clave:}

Participación, património, estratégia, economía, desarrollo

\section{Introducción}

Definición de Liderazgo

"Es el proceso de influencia de líderes y seguidores para alcanzar los objetivos de la organización mediante el cambio". 1

Según Robert N. y Christopher F. (2005) La palabra líder se refiere a alguien que puede ser administrador 0 subordinado, un líder siempre puede influir sobre los demás. 1

"Es el proceso de influir, guiar o dirigir a los miembros del grupo hacia el éxito en la consecución de metas y objetivos organizacionales". 2

Naturaleza de ser Líder.

"Los líderes eficaces no solo nacen con cierta capacidad de liderazgo, sino que además la cultivan, así que cierta habilidad natural puede presentar ciertas ventajas 0 desventajas para un líder". 1

\footnotetext{
a Universidad Autónoma de Tlaxcala, Facultad de Ciencias Económico Administrativas, Contaduría Pública https://orcid.org/0000-00016971-6873, Email: maribel.nava.p@uatx.mx

a Universidad Autónoma de Tlaxcala, Facultad de Ciencias Económico Administrativas, Negocios Internacionales https://orcid.org/00000002-0234-0450, Email: lucero.cuatepotzos@uatx.mx

a Universidad Autónoma de Tlaxcala, Facultad de Ciencias Económico Administrativas, Administración https://orcid.org/0000-0001-64943727, Email: mariadelcarmen.rodriguez@uatx.mx 
Liderazgo en equipos.

Nos señalan Arévalo y Polgatti (2004) el equipo de trabajo permite enfrentar el contexto inevitable del cambio, haciéndose parte de él. Asumir esto, no sólo implica adaptarse a los cambios del entorno, sino que también conformar un grupo humano que constantemente debe decidir quién asume el liderazgo, cómo se apoyan y motivan los integrantes del equipo, como se resuelven los problemas a los que diariamente se ven enfrentados. 3

Los equipos de trabajo pueden ser definidos como: una comunidad de personas que comparten significados y una(s) meta(s) común(es), cuyas acciones son interdependientes y situadas socio históricamente, y en donde el sentido de pertenencia viene dado por la responsabilidad, el compromiso y la confianza que los miembros sienten de forma recíproca.

Krause (2001) menciona una serie de aspectos funcionales que caracterizarían una comunidad saludable y que a nosotros nos parecen perfectamente aplicables a los equipos de trabajo. 4

"En primer lugar, se menciona la existencia de actividades conjuntas que faciliten la integración social". 4

\section{Liderazgo organizacional.}

"Durante las últimas décadas se ha resaltado el papel que juega el cambio en las organizaciones. La turbulencia del entorno, caracterizada por cambios discontinuos e impredecibles, ha contribuido a provocar un cuestionamiento de las premisas utilizadas para entender el funcionamiento organizacional" 5

"Las teorías transaccionales de liderazgo se centran en cómo los líderes pueden motivar a sus seguidores creando intercambios justos y clarificando beneficios y responsabilidades mutuas; las teorías de liderazgo transformacional, por su parte, proponen que el líder debe estimular a sus iguales y seguidores para que consideren sus trabajos desde distintas perspectivas; hacerles conscientes de la misión o visión del equipo y de la organización; facilitar el desarrollo máximo de todo su potencial y motivarlos para que, más allá de sus propios intereses, tengan en cuenta los intereses que benefician al grupo". 6

Liderazgo empresas familiares.
Aquellas empresas familiares que llegan a la segunda e incluso tercera generación, históricamente se han centrado en la gestión del negocio, siendo ahora el momento de tomar conciencia de la importancia que están cobrando otros factores: las nuevas generaciones acceden a la dirección con nuevos puntos de vista que pueden confrontar con la forma en la gestión de la generación anterior o del fundador.

En el Equipo Familiar (22\% de los casos) la complejidad de la empresa es baja y, por el contrario, la de la familia elevada. Coloquialmente podría decirse que "hay mucha familia para poca empresa". Su edad media es de 45 años.

El modelo de gestión de empresa familiar, Modelo de Gestión de Riesgo Estructural 2 que pone en evidencia la necesidad de que las empresas familiares deben estar atentas al desarrollo de su estructura, y de la relación entre las características familiares y de gestión que se están dando en su empresa, para asegurar la perdurabilidad y continuidad del negocio en el futuro.

\section{Empresas Familiares}

El campo de estudio de las empresas familiares se remonta apenas a 1975, cuando el Dr. León Danco publicó su obra pionera, A Guide for the Business Owner and His Family. Si bien es cierto que no son muchas las investigaciones existentes en el campo de las empresas familiares, cabe preguntarse si será realmente relevante el estudio de las mismas y qué tan importante son para la economía de un país. Serna y Suárez (2005) presentan una serie de datos que pueden confirmar la importancia de la participación de las empresas familiares en las economías de diferentes países del mundo, como es el caso de Chile con el 68\% de empresas familiares, Colombia con un $65 \%$, España con un $71 \%$ e Italia con un $99 \%$ de empresas de este tipo. 7

La organización, gerencia, decisiones, continuidad, entre otros, son aspectos que difieren entre las empresas familiares y las organizaciones no familiares.

Como lo señala Leach (1993), las empresas familiares no solo se diferencian en una serie de aspectos de las empresas no familiares, sino que también funcionan de una manera distinta, de ahí que es imprescindible que se conozcan y se puedan comprender estas diferencias para mejorar la gerencia de las mismas. 8

Las empresas familiares coexisten con empresas que no presentan características de familiares, mostrando algunos rasgos que las diferencian entre sí. De igual 
manera, se puede decir que las empresas familiares son numerosas en comparación con las no familiares en la mayoría de los países del mundo.

Las empresas de Venezuela, y particularmente las de Mérida, no escapan a esta realidad. Considerando las características propias de las empresas venezolanas, y dentro de estas, las de las empresas merideñas, resulta de interés en el ámbito de estudio del sector empresarial conocer las características y la forma de gerencia presente en las empresas familiares, dada la importancia de estos negocios en las economías de otros países y regiones del mundo.

El principal rasgo característico de las empresas familiares es la estrecha relación existente en la vida de la empresa -empresa familiar- y la vida de la familia familia empresaria-; o dicho de otro modo, entre propiedad, gestión y familia. La interrelación de estos tres elementos, propiedad, gestión y familia, hacen que estas empresas tengan elementos claramente diferenciados respecto de la empresa no familiar.

De esta interacción, se da un complejo entramado de situaciones representadas en el modelo de tres círculos fruto de las investigaciones de Lansberg (1983), Ward (1991) y Tagiuri y Davis, (1996), entre otros, según el cual la empresa familiar se puede considerar como una organización formada por un conjunto complejo de elementos -familia, propiedad y grupos de gestión-, cada uno de los cuales con sus propias normas de actuación, los cuales se encuentran entrelazados dinámicamente. 9 , $10,11$.

Esta interconexión supone que cualquier cambio en uno de los elementos afecta inevitablemente al resto de sistemas. Además, el modelo de los tres círculos permite identificar los principales conflictos originados por los distintos grupos de interés que intervienen en la empresa familiar.

\section{Conceptos de empresa familiar}

De acuerdo con Leach (2002), en Bañegil Palacios, Barroso Martínez y Tato Jiménez (2011), la empresa familiar es una organización social de gran complejidad, donde conviven la empresa y la familia, entes que se desempeñan como dos subsistemas superpuestos, interdependientes y generadores de conflicto.12, 13.

De acuerdo con Lozano Posso (2000), la empresa familiar también puede definirse como un tipo de unidad empresarial que suele operar a partir de la disponibilidad de capital y trabajo de origen familiar o doméstico, y en la cual las relaciones laborales presentan elementos diferenciadores con respecto a otro tipo de organizaciones empresariales. 14

Soto Maciel (2013), la define como aquella compañía donde la mayoría de los votos está en manos de la familia controladora, incluyendo al fundador, el cual busca heredar la empresa a sus descendientes, obligándolos a conservar las raíces y los valores que permitan mantener la unidad familiar a través del patrimonio. 15

\section{Características}

Así como toda organización tiene una serie de características comunes, las empresas familiares poseen a su vez una serie de características que las hacen únicas y permiten la identificación de las mismas. Existen 3 aspectos considerados en la mayoría de las definiciones sobre empresas familiares que sirven para delimitarlas. Gallo (1995), también coincide con dichos aspectos:

a. La propiedad y control sobre la empresa; en relación con este aspecto, puede citarse el porcentaje de participación en el capital perteneciente a una familia.

b. El poder que la familia ejerce sobre la empresa, normalmente por el trabajo desempeñado por algunos de los miembros de la familia, es decir, la realización de funciones de alta gerencia en la empresa, específicamente la dirección general.

c. La intención de transferir la empresa a generaciones venideras, es decir, mantener en el futuro la participación de la familia en la empresa.

\section{Consejo Familiar. 16}

El Consejo de Familia es la estructura de gobierno que sirve de nexo entre la familia y la empresa, que se constituye como vehículo de encuentro para comunicar los planes de la empresa a la familia, y los de la familia a la empresa.

Esto es, el órgano que regula el funcionamiento de la familia empresarial, y, en especial, de las relaciones de la familia con sus actividades empresariales y extraempresariales ligadas a la empresa $y / 0$ al patrimonio familiar (Amat, 2001). 17

Como principal órgano de la familia empresaria, está integrado por representantes de todas las ramas y generaciones de la familia, con independencia de que trabajen o no en la empresa. 
Debe funcionar como vehículo de comunicación entre los miembros de la familia, que garantice el mantenimiento de la cultura y de los valores familiares en la empresa y en la gestión del patrimonio familiar, además de ser el responsable de implantar y adaptar el protocolo familiar, tratando de buscar consenso entre sus miembros sobre lo que debe estar regulado.

Se trata del órgano idóneo para discutir los planes de los miembros de la familia y para dilucidar sobre las situaciones especiales o extraordinarias en la vida de los distintos familiares, dando solución a sus problemas al margen de la empresa.

A través del consejo se organizan además las reuniones familiares, en las que se enseña y trasmite a los miembros más jóvenes del clan y a los parientes políticos los valores, tradiciones e historia de la familia.

Sus reuniones deben ser periódicas y es deseable que exista un calendario de las mismas previamente fijado en el protocolo familiar, siendo presidido por un miembro de la familia respetado por todos o por uno de los fundadores de la empresa.

\section{Protocolo de Sucesión}

El peso de la Sucesión y la Administración en las EF

Las empresas cuentan con estándares éticos escritos por mínimos que estos sean inherentes a sus culturas, así como sistemas y procesos organizativos (Brenner,1992); la supervivencia de las EF, Steckerl (2006) nos dice que se considerada por muchos expertos como el principal problema, en la segunda y tercera generación se vuelven aún más complejas, y existen mayores problemas al estar integrada por más de una familia y es aún más grave cuando se involucra a parientes terceros o políticos, es decir, de haberse fundado la empresa por su fundador, pasa a ser empresa de muchos familiares, donde todos ellos desean tomar poder sobre ella. 18, 19.

Es importante que el personal que labora en la EF se encuentre motivado, sin embargo, los propios empleados suelen ser familiares, para ello la reorganización también se plantea, es decir, es volver a plantearse objetivos del negocio a corto y largo plazo (Grabinsky, 1992). 20

Una reorganización o reingeniería es crucial para la mejora de decisiones por parte del fundador, la relación entre padres e hijos es necesaria y fundamental para la cooperación y el buen funcionamiento de la empresa, Grabinsky (1992) refieren que la relación entre estos dos, está dada en términos de autoridad y poder, el choque y la confrontación se hallan a la orden del día por ambas partes. 20

Por un lado está el deseo del padre por afirmar su autoridad sobre los miembros de la empresa, pero por otra parte, se encuentra el deseo de los hijos adquirir poder, Ginebra (2001) hace referencia a esto mismo como "el ejercicio de poder que acaba destruyendo a la empresa y también a la familia" y así los problemas van más allá por el poder de la administración que los problemas económicos-financieros, debido a la falta de una estructuración formal y escrita que permita la incorporación de nuevas generaciones a la administración. 21

A falta de una sucesión planificada dentro de la empresa, sabemos que puede causar una confrontación entre los accionistas originando una crisis estructural con consecuencias en las relaciones dentro de la familia bastante crítica por la falta de comunicación e información adecuada entre la empresa y los miembros de la familia, además de presentarse problemas entre fundador e hijos, también existen problemas entre hermanos quienes buscan tomar mayor poder y protección por parte del fundador dentro de la empresa.

Cabe citar una frase por parte de Ayala (2012) que lastimosamente es bastante cierta, las tres " $F$ : el padre la Funda, el hijo la Fortalece y el nieto la Funde", o también "abuelo rico, padre millonario, nieto miserable", del filme mexicano "Nosotros los nobles". 22

Gallo y Luostarinen (1991) nos muestran cómo el ciclo de vida de la empresa familiar puede dificultar un apoyo, por parte de la dirección de la misma, a una estrategia de crecimiento. 16

Por una parte, se señala la influencia que ejerce en este sentido la última etapa de la primera generación, en la que el fundador generalmente se resiste a cambiar la orientación estratégica de la empresa y a rejuvenecer los miembros de la estructura de responsabilidades.

Por otra parte, se hace hincapié en la crisis estructural asociada a la segunda generación en la que, después de prolongados períodos de desacuerdo en la asignación de responsabilidades entre algunos miembros de la familia, se llega a un consenso en el cual se propone no hacer cambios con el fin de que todo se mantenga en las mismas condiciones que cuando dominaba la primera generación. 
De hecho, Begley y Boyd (1986) indican que aquellas empresas dirigidas por el fundador crecen más rápidamente que aquellas dirigidas por el sucesor. 24

Las empresas cuentan con estándares éticos escritos por mínimos que estos sean inherentes a sus culturas, así como sistemas y procesos organizativos (Brenner,1992); la supervivencia de las EF, Steckerl (2006) nos dice que se considerada por muchos expertos como el principal problema, en la segunda y tercera generación se vuelven aún más complejas, y existen mayores problemas al estar integrada por más de una familia y es aún más grave cuando se involucra a parientes terceros o políticos, es decir, de haberse fundado la empresa por su fundador, pasa a ser empresa de muchos familiares, donde todos ellos desean tomar poder sobre ella. 18, 19

Es importante que el personal que labora en la EF se encuentre motivado, sin embargo, los propios empleados suelen ser familiares, para ello la reorganización también se plantea, es decir, es volver a plantearse objetivos del negocio a corto y largo plazo (Grabinsky, 1992). 19

Una reorganización o reingeniería es crucial para la mejora de decisiones por parte del fundador, la relación entre padres e hijos es necesaria y fundamental para la cooperación y el buen funcionamiento de la empresa, Grabinsky (1992) refieren que la relación entre estos dos, está dada en términos de autoridad y poder, el choque y la confrontación se hallan a la orden del día por ambas partes. 19

Por un lado está el deseo del padre por afirmar su autoridad sobre los miembros de la empresa, pero por otra parte, se encuentra el deseo de los hijos adquirir poder, Ginebra (2001) hace referencia a esto mismo como "el ejercicio de poder que acaba destruyendo a la empresa y también a la familia" y así los problemas van más allá por el poder de la administración que los problemas económicos-financieros, debido a la falta de una estructuración formal y escrita que permita la incorporación de nuevas generaciones a la administración. 21

A falta de una sucesión planificada dentro de la empresa, sabemos que puede causar una confrontación entre los accionistas originando una crisis estructural con consecuencias en las relaciones dentro de la familia bastante crítica por la falta de comunicación e información adecuada entre la empresa y los miembros de la familia, además de presentarse problemas entre fundador e hijos, también existen problemas entre hermanos quienes buscan tomar mayor poder y protección por parte del fundador dentro de la empresa.
Cabe citar una frase por parte de Ayala (2012) que lastimosamente es bastante cierta, las tres " $F$ : el padre la Funda, el hijo la Fortalece y el nieto la Funde", o también "abuelo rico, padre millonario, nieto miserable", del filme mexicano "Nosotros los nobles". 22

Gallo y Luostarinen (1991) nos muestran cómo el ciclo de vida de la empresa familiar puede dificultar un apoyo, por parte de la dirección de la misma, a una estrategia de crecimiento. 16

Por una parte, se señala la influencia que ejerce en este sentido la última etapa de la primera generación, en la que el fundador generalmente se resiste a cambiar la orientación estratégica de la empresa y a rejuvenecer los miembros de la estructura de responsabilidades.

Se hace hincapié en la crisis estructural asociada a la segunda generación en la que, después de prolongados períodos de desacuerdo en la asignación de responsabilidades entre algunos miembros de la familia, se llega a un consenso en el cual se propone no hacer cambios con el fin de que todo se mantenga en las mismas condiciones que cuando dominaba la primera generación.

De hecho, Begley y Boyd (1986) indican que aquellas empresas dirigidas por el fundador crecen más rápidamente que aquellas dirigidas por el sucesor. 23

La sucesión, en el ámbito de la teoría de la empresa, se conoce como: «el abuelo la funda, los hijos la debilitan y Ios nietos la entierran» (Le Breton-Miller, Miller \& Steier, 2004; Royer, Simons, Boyd \& Rafferty, 2008 en Soto Maciel, 2013; González, 2010). 24, 25.

Existe una coincidencia unánime de los expertos al señalar que el principal inconveniente de las empresas familiares radica en la dificultad de mantener la continuidad a lo largo de distintas generaciones.

De esta manera, la planificación de la sucesión consiste en establecer una estrategia hereditaria en la que entran en juego las reglas de parentesco. El matrimonio unifica, la herencia separa, la propiedad permanece.

Dentro del marco legal vigente, cada empresa familiar diseña una estrategia hereditaria particular que no puede ser prevista de antemano. Por lo general, en la mayoría de las empresas familiares, la falta de preparación de las generaciones sucesivas para manejar las demandas de una empresa creciente y una familia mucho mayor, genera una gran crisis (Soto Maciel, 2013). 15 
Navarrete (2008, en Soto Maciel, 2013), analiza los factores de carácter familiar, empresarial y de propiedad, que contribuyen a explicar la continuidad de las empresas familiares. Entre los factores de carácter familiar, la autora incluye la influencia de ciertas características de la relación familiar, como la fuerza de la relación en términos de interdependencia, cohesión y adaptabilidad. 15

Entre los factores empresariales menciona la importancia del tiempo que la empresa ha sido propiedad familiar, la manera en que la familia ha participado en su dirección, así como el desarrollo del sucesor y su vinculación con la dinámica empresarial.

Entre los principales obstáculos del proceso de transición generacional, esta misma autora reconoce en el propietario-fundador la resistencia para transferir el poder y el desinterés de los miembros de la familia para participar activamente en cuestiones estratégicas, así como la falta de planificación del proceso de sucesión.

Además de la sucesión de la propiedad, otro de los factores que afectan la falta de continuidad es el escaso nivel de emprendimiento y la falta de gestión profesional. Para McCann, León-Guerrero \& Haley (2011, en Bañegil Palacios, Barroso Martínez \& Tato Jiménez, 2011), profesionalizar el manejo de la empresa es dejar de lado los métodos intuitivos de gestión familiar y adoptar la planificación y el control del crecimiento aplicando métodos estratégicos. 26

La profesionalización en las empresas familiares es la contratación de directivos profesionales no pertenecientes a la familia, que aporten su experiencia y formación en el área concreta que se desea potenciar (Barroso, 2014). 27

\section{Referencias}

[1] Lussier Robert N., Achua Christopher F., Liderazgo 2005; 2da Edición.

[2] Aguera I. Enrique, Liderazgo y Compromiso Social 2004; 1era Edición.

[3] Arévalo y Polgatti Liderazgo y Equipos de Trabajo: Una Nueva Forma de Entender la Dinámica Organizacional 2004; Ciencias Sociales on line. III(1).

[4] Krause Jacob, Hacia una redefinición del concepto de comunidadcuatro ejes para un análisis crítico y una propuesta-, 2001.

[5] (Artacho, Fuentes y Veroz, 2003; Ibisate, 2002; Gosling y Mintzberg, 2003).

[6] Sánchez margarita, La investigació sobre el desarrollo y la enseñanza de las habilidades de pensamiento 2002.

[7] Serna y Suárez, La profecionalización, elemento clave del éxito de la empresa 2005.

[8] Leach P., La empresa familiar 1993.
[9] Lansberg Ivan, Managing human resources in family firms: The problem if institutional overlap 1983.

[10] Ward Linda, Gender and publishing in sociology 1991

[11] Tagiuri Renato; Davis Jhon, Bivalent attributes of the family firm 1996.

[12] Leach, Jhon Research in subject Knowledge and applications 2002.

[13] Bañegil Palacios, Barroso Martínez y Tato Jiménez 2011.

[14] Lozano Posso, El protocolo en las empresas de propiedad familiar 2000 .

[15] Soto Maciel, La empresa familiar en México -Contaduría y Administración 2013.

[16] Gallo, M.A. Empresa familiar: textos y casos 1995.

[17] Amat, J. . La continuidad de la empresa familiar. Barcelona: Gestión. 2001.

[18] Brenner, Steven Programas éticos y sus dimensiones 1992.

[19] Steckerl, Vanessa Modelo explicativo de una empresa familiar que relaciona valores del fundador, cultura organizacional y orientación al mercado 2006

[20] Grabinsky Steider, La empresa familiar 1992.

[21] Ginebra, J. 2001;. Las empresas familiares: su dirección y su continuidad. México: Panorama.

[22] Ayala, G. T. 2012; Profesionalizando la Empresa Familiar. Terra Networks México.

[23] Begley y Boyd, Pshycological traits of rural entrepreneurs 1986.

[24] Le Breton-Miller, Miller \& Steier, Toward an Integrative Model of Effective FOB succession 2004.

[25] González, R. 2010; La continuidad de la empresa familiar. Anuario Jurídico y Económico Escurialense, 401-410.

[26] Bañegil Palacios, Barroso Martínez \& Tato Jiménez, Profesionalizarse, emprender y aliarse para que la empresa familiar continúe 2011.

[27] Barroso, Influencia del liderazgo sobre el clima organizacional 2014

Leach P. La empresa familiar 1993.

(1)

